\title{
Eccentric hypertrophy predicts adverse events in patients undergoing percutaneous coronary intervention for acute coronary syndrome
}

Edward T. Ha ${ }^{1}$, Marc Cohen ${ }^{2,3}$, Stephen J. Peterson ${ }^{1,4}$, Wilbert S. Aronow ${ }^{5,6}$

\begin{abstract}
1Department of Internal Medicine, New York-Presbyterian Brooklyn Methodist Hospital, Brooklyn, NY, USA

2Department of Cardiology, Newark Beth Israel Medical Center, Newark, NJ, USA ${ }^{3}$ Department of Medicine, Rutgers-New Jersey Medical School, Newark, NJ, USA ${ }^{4}$ Department of Medicine, Weill Cornell Medical College, New York, NY

${ }^{5}$ Department of Medicine, New York Medical College, Valhalla, NY, USA

${ }^{6}$ Department of Cardiology, Westchester Medical Center, Valhalla, NY, USA
\end{abstract}

Submitted: 4 February 2021

Accepted: 8 February 2021

Arch Med Sci Atheroscler Dis 2021; 6: e21-e27

DOI: https://doi.org/10.5114/amsad.2021.105175

Copyright $\odot 2021$ Termedia \& Banach

\section{Abstract}

Introduction: The effect of the type of left ventricular hypertrophy in patients presenting with an acute coronary syndrome (ACS) on long-term outcomes is ill-defined. The purpose of this study was to investigate the prognostic effect of concentric $(\mathrm{CH})$ or eccentric hypertrophy $(\mathrm{EH})$ on adverse outcomes in patients presenting with ACS undergoing percutaneous coronary intervention $(\mathrm{PCl})$.

Material and methods: We analyzed 1-year follow-up data from a single-institution, retrospective, observational study that enrolled 1,153 patients who presented with ACS and were treated with $\mathrm{PCI}$, for whom echocardiographic data were available.

Results: Normal geometry was observed in 718 (62.3\%) patients, while $27.2 \%$ had $\mathrm{CH}$ and $10.5 \%$ had $\mathrm{EH}$. The primary endpoint of all-cause death $(n=90,7.8 \%)$ occurred in $6.4 \%, 8.0 \%$, and $14.9 \%$ of patients with no, concentric, or eccentric hypertrophy, respectively $(p=0.005)$. Major adverse cardiac events (MACE - all-cause death, non-fatal myocardial infarction or stroke or hospitalization for bleeding) occurred in $13.9 \%, 17.8 \%, 30.6 \%$, respectively $(p<0.001)$. Age (HR per year $=1.04(1.02,1.05), p<0.001)$, female gender $(H R=1.56(1.12,2.16), p=0.008)$, diabetes $(H R=1.49(1.07$, 2.06), $p=0.02)$, eccentric hypertrophy ( $H R=1.58(1.006,2.47), p=0.047)$, peak troponin I (HR per $1 \mathrm{ng} / \mathrm{ml}=1.004(1.001,1.006), p=0.004)$ and left ventricular ejection fraction $<50 \%(H R=1.57(1.12,2.20), p<0.008)$ were significant predictors of MACE.

Conclusions: The presence of eccentric hypertrophy in ACS patients undergoing $\mathrm{PCl}$ is an independent predictor of adverse outcomes at 1 year.

Key words: heart failure, myocardial infarction, left ventricular hypertrophy, major adverse cardiac events, heart attack.

\section{Introduction}

Coronary artery disease (CAD) is a leading cause of death in the developed world [1]. Acute plaque rupture and thrombosis causes acute coronary syndrome (ACS) and is frequently treated with percutaneous coronary intervention $(\mathrm{PCl})$. Increasing age, lower left ventricular ejec-

\author{
Corresponding author: \\ Edward T. Ha MD, MSc \\ Department of \\ Internal Medicine \\ New York-Presbyterian \\ Brooklyn Methodist \\ Hospital \\ $5066^{\text {th }}$ Street \\ Brooklyn, 11215 \\ NY, USA \\ Phone: 718-780-3000 \\ E-mail: edh9028@nyp.org
}


tion fraction (LVEF), and infarct size have all been linked with worse outcomes [2].

Prior studies have shown left ventricular hypertrophy (LVH) to be an independent predictor of worse outcomes in those with hypertension and CAD [3-5]. Less clear are its implications in ACS [6-10]. LVH is a heterogeneous classification and can be subdivided into concentric $(\mathrm{CH})$ and eccentric hypertrophy $(\mathrm{EH})$. Recently, human epidemiologic studies have supported animal data indicating that LV geometry typically progresses from normal to $\mathrm{CH}$, and then to $\mathrm{EH}$, eventually leading to decompensated heart failure [11, 12]. Furthermore, recent studies have shown differential clinical and biomarker phenotypes between $\mathrm{CH}$ and $\mathrm{EH}$ in those with heart failure with reduced ejection fraction (HFrEF), which indicates that $\mathrm{LVH}$ may be a clinical over-simplification [13].

The purpose of this study was to investigate the prognostic effect of concentric and eccentric hypertrophy on adverse outcomes in patients presenting with ACS undergoing $\mathrm{PCl}$.

\section{Material and methods}

We performed an observational study of patients presenting to an urban, community hospital with ACS who underwent $\mathrm{PCl}$ and were discharged alive between September 23, 2011 and July 31,2017 . The follow-up period of the primary study was 1 year. Qualified patients were identified from an institutional registry that enrolled all patients undergoing PCI for mandatory reporting to the New York State Department of Health (NYSDOH) and is exempt from the need for patient consent if de-identification of data for reporting is maintained. We excluded PCl patients who did not have ACS, based on clinical presentation, elevation of cardiac enzymes (troponin I and/or CK-MB) or ST-segment changes prior to the procedure. Peak troponin I (ng/ml) obtained during the hospital stay was abstracted from medical records. Transthoracic echocardiogram was performed during the index hospitalization utilizing a protocol in line with the American Society of Echocardiography/Intersocietal Accreditation Commission and it was interpreted by a board-certified cardiologist. The 2D measurements of left ventricular end diastolic diameter (LVEDd), intraventricular septal diameter (IVS), and posterior wall diameter (PWD) were taken from parasternal long axis views. LV mass index (LVMI) was calculated according to the Devereux equation normalized to body surface area [14]:

$L V M I\left(\frac{\mathrm{g}}{\mathrm{m}^{2}}\right)=\frac{0.8\left\{1.04\left[\left([L V E D D+I V S d+P W d]^{3}-L V E D D^{3}\right)\right]\right\}+0.6}{\text { body surface area }}$

Relative wall thickness (RWT) was calculated as:

$$
R W T=\frac{2 \times P W d}{L V E D d}
$$

In accordance with American Society of Echocardiography (ASE) guidelines, an LVMI $\geq 95$ for females and $\geq 115 \mathrm{~g} / \mathrm{m}^{2}$ for males was suggestive of LVH, whereas an RWT $\geq 0.42$ was suggestive of concentric adaptations [15]. Four phenotypes of cardiac geometry were identified: normal, concentric remodeling (grouped together as normal geometry), concentric hypertrophy (defined as LVMI $\geq 95$ and RWT $\geq 0.42$ ) and eccentric hypertrophy (defined as LVMI $\geq 95$ and RWT $\leq 0.42$ ). Left ventricular ejection fraction (LVEF) was measured using the 2D, bi-plane or single plane method of disks at the discretion of the interpreting cardiologist. The primary endpoint was all-cause mortality within 1 year after hospital discharge, which was verified by reviewing medical records or by contacting primary care physicians or the patients' families, when necessary. The secondary endpoint was major adverse cardiac events (MACE) after discharge, which was defined as a composite of all-cause mortality, non-fatal myocardial infarction, non-fatal stroke, or hospitalization for bleeding, adjudicated based on primary discharge diagnosis and verified in a similar fashion.

\section{Statistical analysis}

Continuous and categorical variables are presented as mean with one standard deviation or median with interquartile range, as appropriate, and were compared with one-way ANOVA for means, Kruskal-Wallis ANOVA for medians, and $\chi^{2}$ test for proportions. Event rates were estimated using the Kaplan-Meier time-to-event methodology and compared using log-rank tests. Multivariable Cox proportional hazard regression was used to determine the independent predictors of the primary and secondary outcomes. The following demographic and clinical covariates were simultaneously included in the model for the primary and secondary outcomes: age, gender, hypertension, diabetes, prior myocardial infarction (MI), baseline creatinine, LVEF $<50 \%$, peak troponin I, normal geometry, concentric hypertrophy, and eccentric hypertrophy. Two dummy variables were used to include the three groups of cardiac geometries simultaneously with reported hazard ratios $(\mathrm{HR})$ of $\mathrm{CH}$ and $\mathrm{EH}$ in reference to a $\mathrm{HR}$ of 1 for normal geometry. The significance level was set at $p<0.05$ (two-sided). All analyses were performed with IBM SPSS Statistics for Mac, version 24 (IBM Corp., Armonk, N.Y., USA).

\section{Results}

Between September 2011 and July 2017, 1,610 ACS patients were identified in the institutional registry who underwent $\mathrm{PCl}$. Vital status could not be established in 165 patients. An echocardiogram was not performed for 292 patients. A total of 
1,153 patients were enrolled in the study. Baseline characteristics, by cardiac geometry, are displayed in Table I. In summary, patients with normal geometry tended to be younger, male and have fewer comorbidities, but more likely to present with ST-elevation myocardial infarction (STEMI).

The incidence of adverse events in follow-up is shown in Table II. Eccentric hypertrophy was associated with an increased incidence of allcause death and non-fatal MI when compared to normal or concentric hypertrophy $(p=0.007$ and $p<0.001$, respectively).
The primary endpoint of all-cause death $(n=90$, $7.8 \%$ ) occurred in $6.5 \%, 8.0 \%$, and $14.9 \%$ of patients with no, concentric, and eccentric hypertrophy, respectively $(p=0.006)$ (Figure 1$)$.

In multivariable Cox regression analysis, age (hazard ratio $(H R$ (per year) $=1.07(1.04,1.09)$, $p<0.001))$, female gender $(H R=1.64$ (1.009, 2.65), $p=0.046)$, baseline creatinine (HR per $1 \mathrm{mg} / \mathrm{dl})=1.12(1.02,1.23), p=0.02)$ and LVEF $<50 \%(H R=2.39(1.44,3.95), p<0.001)$, and peak troponin I ((HR per $1 \mathrm{ng} / \mathrm{ml})=1.003(1.02,1.23)$, $p=0.04)$ ) were significant predictors of death,

Table I. Comparative table of baseline demographics of various cardiac geometries

\begin{tabular}{|c|c|c|c|c|}
\hline Parameter & $\begin{array}{c}\text { Normal geometry } \\
(n=718)\end{array}$ & $\begin{array}{l}\text { Concentric } \\
\text { hypertrophy } \\
(n=314)\end{array}$ & $\begin{array}{c}\text { Eccentric } \\
\text { hypertrophy } \\
(n=121)\end{array}$ & $P$-value \\
\hline \multicolumn{5}{|l|}{ Demographic data: } \\
\hline Age & $65.7 \pm 20$ & $66.9 \pm 16.5$ & $68.7 \pm 18.7$ & 0.01 \\
\hline Male gender & $464(67)$ & $172(55)$ & $58(48)$ & $<0.001$ \\
\hline \multicolumn{5}{|l|}{ Race: } \\
\hline White & $421(59)$ & $159(51)$ & $70(58)$ & 0.054 \\
\hline Black & $236(33)$ & $138(44)$ & $43(36)$ & 0.002 \\
\hline BMI & $28.4 \pm 7.2$ & $28.8 \pm 7.1$ & $27.6 \pm 7.4$ & 0.146 \\
\hline Hypertension & $583(81)$ & $292(93)$ & $110(91)$ & $<0.001$ \\
\hline Dyslipidemia & $538(75)$ & $246(78)$ & $97(80)$ & 0.29 \\
\hline Diabetes & $317(44)$ & $171(54)$ & $66(55)$ & 0.003 \\
\hline Smoker & $168(23)$ & $87(28)$ & $26(21)$ & 0.24 \\
\hline FamHx of CAD & $138(19)$ & $51(16)$ & $19(16)$ & 0.40 \\
\hline Current dialysis & $23(3.2)$ & $44(14)$ & $13(11)$ & $<0.001$ \\
\hline Chronic lung disease & $43(6)$ & $24(8)$ & $10(8)$ & 0.47 \\
\hline Prior MI & $137(19)$ & $88(28)$ & $35(29)$ & 0.001 \\
\hline Prior HF & $62(9)$ & $87(28)$ & $43(36)$ & $<0.001$ \\
\hline Prior CVD & $68(9)$ & $42(13)$ & $17(14)$ & 0.09 \\
\hline Prior PAD & $55(8)$ & $22(7)$ & $17(14)$ & 0.04 \\
\hline Prior valve Sx & $5(0.6)$ & $8(3)$ & $1(0.8)$ & 0.04 \\
\hline Prior $\mathrm{PCl}$ & $197(27)$ & $143(46)$ & $63(52)$ & $<0.001$ \\
\hline Prior $C A B G$ & $48(7)$ & $30(10)$ & $20(17)$ & 0.001 \\
\hline Prior cardiogenic shock & $17(2)$ & $2(0.6)$ & $1(0.8)$ & 0.10 \\
\hline Prior cardiac arrest & $16(2)$ & $5(2)$ & $2(2)$ & 0.76 \\
\hline STEMI & $383(53)$ & $116(37)$ & $54(45)$ & $<0.001$ \\
\hline \multicolumn{5}{|c|}{ Echocardiographic and laboratory data: } \\
\hline IVS [cm] & $1.05 \pm 0.3$ & $1.4 \pm 0.3$ & $1.09 \pm 0.2$ & $<0.001$ \\
\hline $\operatorname{LVID}_{\mathrm{d}}[\mathrm{cm}]$ & $4.4 \pm 0.8$ & $4.8 \pm 0.9$ & $5.7 \pm 0.7$ & $<0.001$ \\
\hline PWD [cm] & $1.03 \pm 0.2$ & $1.34 \pm 0.2$ & $1.0 \pm 0.2$ & $<0.001$ \\
\hline RWT & $0.47 \pm 0.15$ & $0.57 \pm 0.1$ & $0.4 \pm 0.1$ & $<0.001$ \\
\hline LVMI & $82.7 \pm 24$ & $137.7 \pm 36$ & $130.1 \pm 31$ & $<0.001$ \\
\hline LVEF & $51.9 \pm 18$ & $47.8 \pm 25$ & $39.2 \pm 30$ & $<0.001$ \\
\hline Peak troponin I [ng/ml] & $5.8 \pm 29.8$ & $3.0 \pm 26.0$ & $4.8 \pm 24.3$ & 0.06 \\
\hline
\end{tabular}


Table II. Incidence of major adverse cardiac events by cardiac geometry

\begin{tabular}{|c|c|c|c|c|}
\hline Parameter & $\begin{array}{l}\text { Normal geometry } \\
\quad(n=718)\end{array}$ & $\begin{array}{c}\text { Concentric LVH } \\
\quad(n=314)\end{array}$ & $\begin{array}{l}\text { Eccentric LVH } \\
\quad(n=121)\end{array}$ & $P$-value \\
\hline All-cause death $(n=90)$ & $47(6.5)$ & $25(8.0)$ & $18(14.9)$ & 0.007 \\
\hline Non-fatal MI $(n=65)$ & $30(4.2)$ & $17(5.4)$ & $18(14.9)$ & $<0.001$ \\
\hline Non-fatal stroke $(n=8)$ & $6(0.008)$ & $1(0.003)$ & $1(0.008)$ & 0.64 \\
\hline Hospitalization for bleeding & $25(3.5)$ & $16(5.1)$ & $8(6.6)$ & 0.20 \\
\hline
\end{tabular}

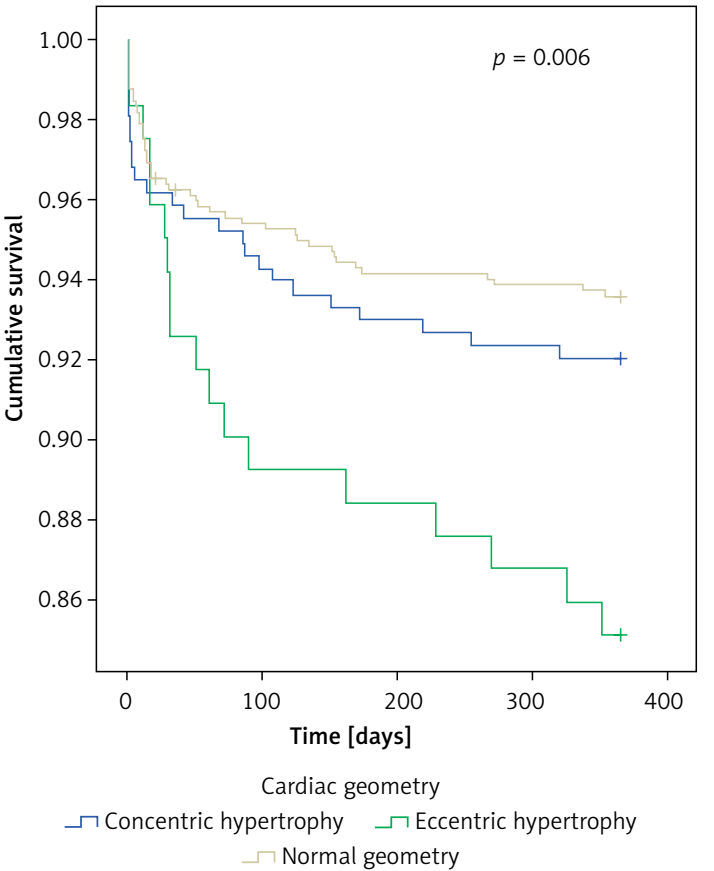

Figure 1. All-cause mortality for various cardiac geometries

while hypertension, diabetes, prior MI, peak troponin I, concentric and eccentric hypertrophy were not (Table III).

Major adverse cardiac events (MACE) occurred in $13.9 \%, 17.8 \%, 30.6 \%$ respectively $(p<0.001$ ) (Figure 2). In multivariable regression analysis, age $(($ HR per year $)=1.04(1.02,1.05), p<0.001)$, female gender $(\mathrm{HR}=1.56(1.12,2.16), p=0.008)$, diabetes $(H R=1.49(1.07,2.06), p=0.02)$, baseline creatinine $((\mathrm{HR}$ per $1 \mathrm{mg} / \mathrm{dl})=1.10(1.04,1.18)$, $p=0.002)$, eccentric hypertrophy $(\mathrm{HR}=1.58(1.006$, 2.47), $p=0.047)$, peak troponin I ((HR per $1 \mathrm{ng} / \mathrm{ml})$ $=1.004(1.001,1.006), p=0.004)$ and LVEF $<50 \%$ $(H R=1.57(1.12,2.20), p<0.008)$ were significant predictors of MACE, while hypertension, prior MI, and concentric hypertrophy were not (Table III).

\section{Discussion}

The major findings from this single-institution, retrospective, observational study of 2D echocardiographic data from 1,153 patients with an index ACS treated with $\mathrm{PCl}$ are as follows: 1) eccentric hypertrophy, but not all LVH, was an independent predictor of MACE at 1 year, after adjusting for age, gender, and co-morbidities such as diabetes CKD, or depressed LVEF. 2) The increased rate of MACE in the EH patients was primarily driven by an increased incidence of all-cause death and non-fatal MI.

Prior studies have largely reported no effect of LVH on outcomes in STEMI $[2,7,8,10]$. Our study cohort consisted of a more real-world representation of ACS patients (nearly half were STEMI patients) and considered $\mathrm{CH}$ and $\mathrm{EH}$ separately, which may partly account for the discrepancy in findings with prior studies. Interestingly, LVH was found to be a risk factor for cardiac death in a single 8-year study, but not total death, acute MI, MACE, or need for revascularization [8]. However, it is not clear whether this increased risk was due to $\mathrm{CH}$ and/or $\mathrm{EH}$. Our finding that eccentric hypertrophy, and not concentric hypertrophy, is associated with worse outcomes in ACS patients undergoing $\mathrm{PCl}$ is novel, as this adverse effect on outcomes is not replicated in HFrEF not experiencing ACS [13].

In the current paradigm, $\mathrm{LVH}$ is thought to be a response to increased cardiac wall stress caused by pressure or volume overload during systole (concentric) and diastole (eccentric), respectively [16]. Increased cardiac wall stress causes hypertrophy and addition of cardiac myocytes in an effort to reduce wall stress. This morphologic change is facilitated by cardiac remodeling - a complex interaction of genetic, molecular, cellular, and neuro-hormonal changes, ultimately affecting cardiac function and contractibility and eventually causing dilated and decompensated heart failure, cardiac dysfunction, arrhythmia, and myocardial infarction (Figure 3, blue pathway) [11, 12].

Alternatively, myocardial infarction compromises cardiac function due to myocyte cell death and necrosis, leading to reduced cardiac function. This acute reduction in cardiac function and inability to keep up with baseline preload causes increased cardiac wall stress, driving cardiac remodeling associated with the development of eccentric hypertrophy (Figure 3, red pathway) [17]. Further, myocardial infarction may cause progression of concentric hypertrophy to eccentric hypertrophy [18]. Thus, the presence of eccentric hypertrophy may identify a high-risk group in patients with CAD associated with worse outcomes following ACS [19]. 
Table III. Independent predictors of 1-year all-cause mortality and major adverse clinical events

\begin{tabular}{|lcc|}
\hline Parameter & HR $(95 \% \mathrm{Cl})$ & P-value \\
\hline All-cause mortality: & $1.07(1.04,1.09)$ & $<0.001$ \\
\hline Age & $1.64(1.009,2.65)$ & 0.046 \\
\hline Gender & $6.73(0.91,49.6)$ & 0.06 \\
\hline Hypertension & $1.55(0.96,2.50)$ & 0.07 \\
\hline Diabetes & $0.98(0.59,1.65)$ & 0.95 \\
\hline Prior MI & $1.12(1.02,1.23)$ & 0.02 \\
\hline Baseline creatinine & $2.39(1.44,3.95)$ & 0.001 \\
\hline LVEF < 50\% & $1.003(1.02,1.23)$ & 0.04 \\
\hline Highest troponin I & 1 & 0.43 \\
\hline Normal geometry (reference) & $0.80(0.47,1.38)$ & 0.83 \\
\hline Concentric hypertrophy & $1.08(0.55,2.09)$ & $<0.001$ \\
\hline Eccentric hypertrophy & $1.04(1.02,1.05)$ & 0.008 \\
\hline MACE: & $1.56(1.12,2.16)$ & 0.95 \\
\hline Age & $1.02(0.57,1.81)$ & 0.02 \\
\hline Gender & $1.49(1.07,2.06)$ & 0.58 \\
\hline Hypertension & $1.11(0.78,1.58)$ & 0.002 \\
\hline Diabetes & $1.10(1.04,1.18)$ & 0.008 \\
\hline Prior MI & $1.57(1.12,2.20)$ & 0.004 \\
\hline Baseline creatinine & $1.004(1.001,1.006)$ & \\
\hline LVEF < 50\% & $1.576(1.006,2.470)$ & \\
\hline Highest troponin I & & \\
\hline Normal geometry (reference) & & \\
\hline Concentric LVH & & \\
\hline Eccentric hypertrophy & & \\
\hline
\end{tabular}

LVEF - left ventricular ejection fraction, $L V H$ - left ventricular hypertrophy, MACE - major adverse cardiac events; defined as cardiovascular death, non-fatal myocardial infarction, non-fatal stroke, and hospitalization for bleeding, MI-myocardial infarction.

According to the Laplace equation, wall stress is proportional to the chamber pressure and radius and inversely proportional to the thickness of the chamber wall. Eccentric hypertrophy is, by definition, characterized by an increase in chamber radius and decrease in chamber wall thickness; thus the wall stress is elevated compared to normal cardiac geometry and $\mathrm{CH}$, as was confirmed in a secondary study of the LIFE trial [20]. This may increase the incidence of all-cause death, as the higher wall stress may predispose to decompensated heart failure and cardiogenic shock (Figure 3). The pathophysiologic mechanism by which $\mathrm{EH}$ increases the incidence of non-fatal MI may be due to the effects of increased myocardial oxygen demand in sensitizing LVH hearts to MI when coronary blood flow is acutely compromised [21, 22]. Indeed, studies have shown the EH phenotype to have the highest myocardial oxygen requirements, when compared to all other cardiac geometries, as measured by the 'triple equation' - left ventricular mass $\times$ heart rate $\times$ left ventricular wall stress [20]. Prior studies have also demonstrated that wall tension is linearly associated with myocardial

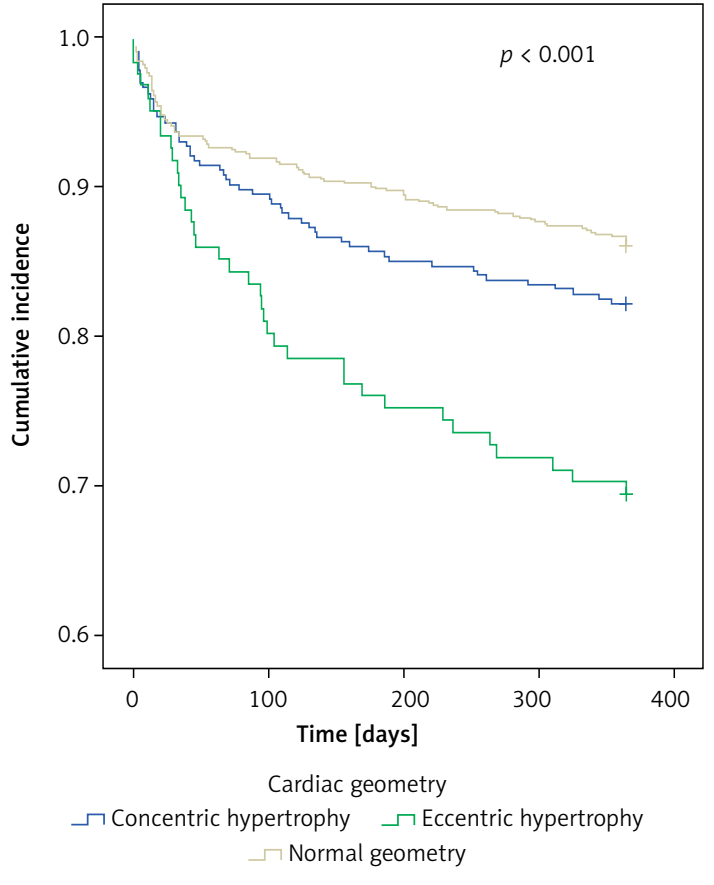

Figure 2. Major adverse cardiac events for various cardiac geometries 


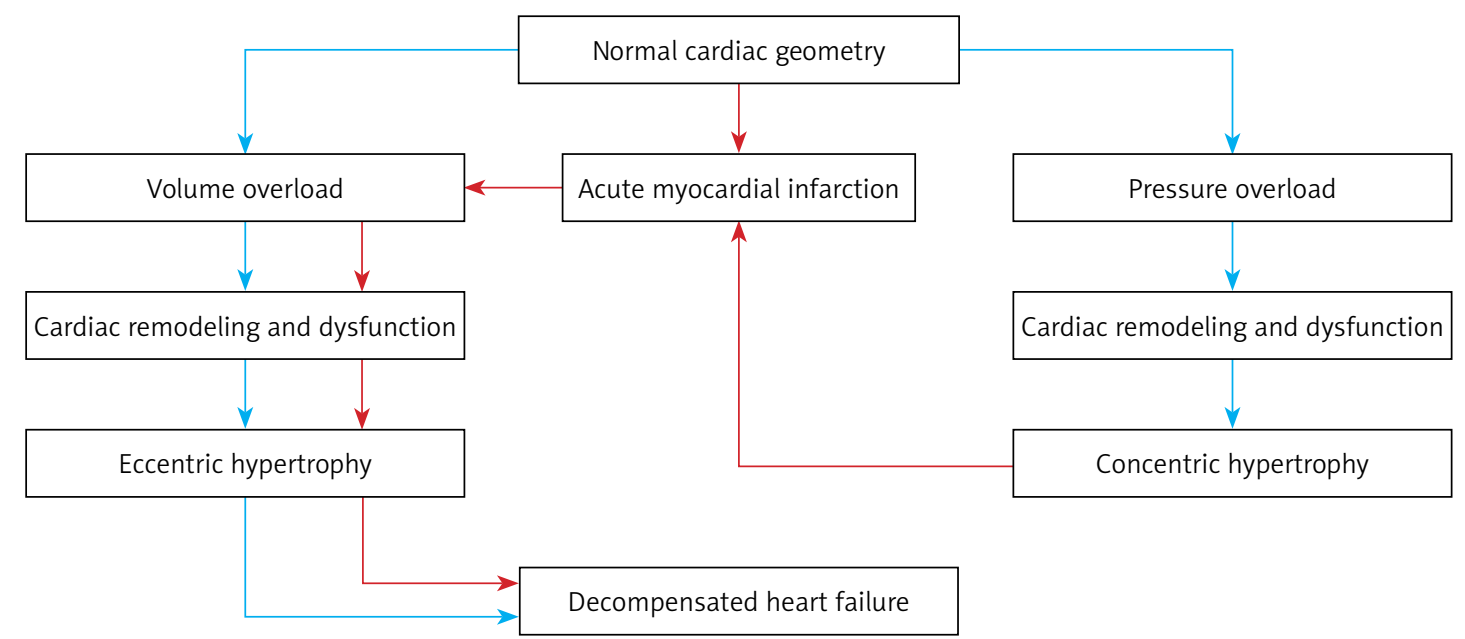

Figure 3. Development and progression to decompensated heart failure according to various stressors

oxygen requirements [23]. Thus, EH patients are at the highest risk of recurrent $\mathrm{MI}$ due to higher myocardial oxygen requirements in the setting of acute coronary thrombosis.

It is not currently known whether EH predisposes to further disease progression and whether this process is partly responsible for the increased incidence of non-fatal $\mathrm{MI}$ following $\mathrm{PCl}$. There is early evidence that junctional adhesion molecule A (JAM-A) accelerates atherosclerotic disease progression in animal models, and JAM-A was found to be a central protein hub in network analyses of protein-protein interactions of circulating biomarkers upregulated in HFrEF with $\mathrm{EH}[13,24]$. Future studies may focus on whether $\mathrm{EH}$ may function as a predictive variable, as evidence exists that the $\mathrm{EH}$ phenotype is reversible $[12,25]$.

We recognize important limitations to our study design, observations and conclusions. The first is the relatively small cohort of patients included in this single-center study when compared to multi-centered randomized clinical trials, which may not be generalizable to the US population as a whole. Second, data were collected retrospectively from the electronic medical records, and were not recorded in a standardized and systematic manner, thus obscuring confounders that cannot be optimally controlled for.

In conclusion, in the present single-institution, retrospective, observational study, in which 2D echocardiographic data from 1,153 patients with an index ACS treated with $\mathrm{PCl}$ were analyzed, after adjustment for important baseline demographic and clinical variables, eccentric hypertrophy was associated with a higher rate of MACE compared to other cardiac geometries, driven by higher mortality and recurrent MI. Further studies are needed to explore the predictive value of this novel clinical variable.

\section{Conflict of interest}

The authors declare no conflict of interest.

\section{References}

1. Virani SS, Alonso A, Benjamin EJ, et al. Heart disease and stroke statistics - 2020 update: a report from the American Heart Association. Circulation 2020; 141: e139-596.

2. Nepper-Christensen L, Lønborg J, Ahtarovski KA, et al. Left ventricular hypertrophy is associated with increased infarct size and decreased myocardial salvage in patients with ST-segment elevation myocardial infarction undergoing primary percutaneous coronary intervention. J Am Heart Assoc 2017; 6: e004823.

3. East MA, Jollis JG, Nelson CL, Marks D, Peterson ED. The influence of left ventricular hypertrophy on survival in patients with coronary artery disease: do race and gender matter? J Am Coll Cardiol 2003; 41: 949-54.

4. Aronow WS, Epstein S, Koenigsberg M. Usefulness of echocardiographic left ventricular hypertrophy and silent ischemia in predicting new cardiac events in elderly patients with systemic hypertension or coronary artery disease. Angiology 1990; 41: 189-93.

5. Koren MJ, Devereux RB, Casale PN, Savage DD, Laragh $J H$. Relation of left ventricular mass and geometry to morbidity and mortality in uncomplicated essential hypertension. Ann Intern Med 1991; 114: 345-52.

6. Haider AW, Larson MG, Benjamin EJ, Levy D. Increased left ventricular mass and hypertrophy are associated with increased risk for sudden death. J Am Coll Cardiol 1998; 32: 1454-9.

7. Stiermaier T, Pöss J, Eitel C, et al. Impact of left ventricular hypertrophy on myocardial injury in patients with ST-segment elevation myocardial infarction. Clin Res Cardiol 2018; 107: 1013-20.

8. Kim YH, Her AY, Choi BG, et al. Impact of left ventricular hypertrophy on longterm clinical outcomes in hypertensive patients who underwent successful percutaneous coronary intervention with drug-eluting stents. Medicine 2018; 97: e12067.

9. Kosmidou I, Leon MB, Zhang Y, et al. Long-term outcomes in women and men following percutaneous coronary intervention. J Am Coll Cardiol 2020; 75: 1631-40. 
10. Brown DL. Effect of left ventricular hypertrophy on longterm survival of patients with coronary artery disease following percutaneous coronary intervention. Heart Int 2009; 4: e9.

11. Inoko M, Kihara Y, Morii I, Fujiwara H, Sasayama S. Transition from compensatory hypertrophy to dilated, failing left ventricles in Dahl salt-sensitive rats. Am J Physiol 1994; 267: H2471-82.

12. Lieb W, Gona P, Larson MG, et al. The natural history of left ventricular geometry in the community: clinical correlates and prognostic significance of change in LV geometric pattern. JACC Cardiovasc Imaging 2014; 7: 870-8.

13. Nauta JF, Hummel YM, Tromp J, et al. Concentric vs. eccentric remodelling in heart failure with reduced ejection fraction: clinical characteristics, pathophysiology and response to treatment. Eur J Heart Fail 2020; 22: 1147-55.

14. Devereux RB, Alonso DR, Lutas EM, et al. Echocardiographic assessment of left ventricular hypertrophy: comparison to necropsy findings. Am J Cardiol 1986; 57: 450-8.

15. Lang RM, Badano LP, Victor MA, et al. Recommendations for cardiac chamber quantification by echocardiography in adults: an update from the American Society of Echocardiography and the European Association of Cardiovascular Imaging. J Am Soc Echocardiogr 2015; 28: 1-39.e14.

16. Grossman W, Jones D, McLaurin LP. Wall stress and patterns of hypertrophy in the human left ventricle. J Clin Invest 1975; 56: 56-64.

17. Peterson KL. Pressure overload hypertrophy and congestive heart failure: Where is the "Achilles" Heel"?" J Am Coll Cardiol 2002; 39: 672-5.

18. Simone G De. Concentric or eccentric hypertrophy: how clinically relevant is the difference? Hypertension 2004; 43: 714-5.

19. Zabalgoitia M, Berning J, Koren MJ, et al. Impact of coronary artery disease on left ventricular systolic function and geometry in hypertensive patients with left ventricular hypertrophy (The LIFE Study). Am J Cardiol 2001; 88: 646-50.

20. Devereux RB, Roman MJ, Palmieri V, et al. Left ventricular wall stresses and wall stress-mass-heart rate products in hypertensive patients with electrocardiographic left ventricular hypertrophy: the LIFE. J Hypertens 2000; 18: 1129-38.

21. Koyanagi S, Eastham CL, Harrison DG, Marcus ML. Increased size of myocardial infarction in dogs with chronic hypertension and left ventricular hypertrophy. Circ Res 1982; 50: 55-62.

22. Dellsperger KC, Clothier JL, Hartnett JA, Haun LM, Marcus ML. Acceleration of the wavefront of myocardial necrosis by chronic hypertension and left ventricular hypertrohpy in dogs. Circ Res 1988; 63: 87-96.

23. Strauer BE. Myocardial oxygen consumption in chronic heart disease: role of wall stress, hypertrophy and coronary reserve. Am J Cardiol 1979; 44: 730-40.

24. Karshovska E, Zhao Z, Blanchet X, et al. Hyperreactivity of junctional adhesion molecule A-deficient platelets accelerates atherosclerosis in hyperlipidemic mice. Circ Res 2015; 116: 587-99.

25. Wachtell K, Dahlöf B, Rokkedal J, et al. Change of left ventricular geometric pattern after 1 year of antihypertensive treatment: the Losartan Intervention For Endpoint reduction in hypertension (LIFE) study. Am Heart J 2002; 144: 1057-64. 